\title{
Abstract: 21 \\ Physical activity as heart failure therapy: patient perspectives
}

Authors:

S. Tierney ${ }^{1}$, C. Deaton ${ }^{1}$, H. Elwers ${ }^{2}$, C. Sange ${ }^{1}$, M. Mamas ${ }^{3}$, M. Rutter ${ }^{3}$, M. Gibson ${ }^{1}$, L. Neyses ${ }^{3},{ }^{1}$ University of Manchester Manchester - United Kingdom, ${ }^{2}$ University Hospitals of South Manchester NHS Foundation Trust - Manchester - United Kingdom, ${ }^{3}$ Central Manchester University Hospitals NHS Foundation Trust - Manchester - United Kingdom,

Topic(s):

Heart failure

\section{Citation:}

European Journal of Cardiovascular Nursing ( 2011 ) 10 ( Supplement 1 ), S26

Purpose: Research has highlighted the benefits of physical activity for people with stable heart failure (HF) in improving mortality, morbidity and quality of life. However, adherence is low and there is little information on patients' perspectives of activity and exercise; no previous qualitative studies were found that focused on the topic, so this investigation aimed to explore patients' views of and beliefs regarding activity.

Methods: This qualitative study involved patients attending 3 UK HF clinics. A purposive sampling approach was adopted to provide maximum variation in terms of gender, age, New York Heart Association (NYHA) class, duration of HF and activity levels. Semi-structured interviews were conducted via telephone. These were recorded and transcribed verbatim and interpreted using framework analysis.

Results: 22 patients ( 7 female) were interviewed. Their mean age was 68.9 years (SD 8.1) and they spanned NYHA classifications I-III. Participants' activity levels were shaped by a) beliefs about benefits or negative impacts of exercise and personal abilities followed by b) support or constraint from others (professionals, family); c) past experience of activity; d) structural barriers (costs, travel, environment). Barriers included co-morbid conditions, symptoms and fear of overdoing it (especially if they had an implantable cardioverter defibrillator). Although some participants enjoyed supervised programmes, every day activities (e.g. gardening) or those with a social element (e.g. dancing, bowling) were generally favoured over formal exercise. Walking was the most frequent activity, albeit hindered by weather and terrain. Enhanced well-being was often cited as a reason to exercise, but few individuals mentioned improvement in symptoms or functional ability; patients did not seem to view exercise as beneficial for their hearts, but linked its importance to generic health promotion messages.

Conclusions: Results from the study suggest areas to incorporate into interventions aimed at promoting the uptake and maintenance of activity among people with HF. Many barriers could be addressed by considering beliefs, providing specific information and feedback regarding benefits, developing innovative programmes that provide greater patient choice, and strategies to overcome structural barriers. Social cognition models (e.g. the Theory of Planned Behaviour) may be of particular use given their emphasis on beliefs, attitudes and confidence about performing behaviours in a social context. 